\title{
Análise do gasto com judicialização de medicamentos no Distrito Federal, Brasil
}

\author{
Analysis of drug expenditure with judicialization in the Federal District, Brazil
}

Análisis del gasto con la judicialización de medicamentos en el Districto Federal, Brasil

Everton Macêdo Silva ${ }^{1}$

Keyla Caroline de Almeida ${ }^{2}$

Glaucia Silveira Carvalho Pessôa ${ }^{3}$

RESUMO: A judicialização como fenômeno de garantia do direito social à saúde é uma questão com discussão crescente no Brasil, devido à definição constitucional de saúde no país, que contempla a integralidade. Objetivo: Analisar o perfil do gasto da saúde pública no Distrito Federal com medicamentos não-padronizados, a fim de compreender quais as circunstâncias em que a judicialização de medicamentos ocorre nesse local. Métodos: Análise jurisprudencial nos processos judiciais sobre medicamentos. Foram utilizados registros administrativos de distribuição dos medicamentos no período de setembro/2014 a agosto/2016. O gasto apurado foi classificado por item e por grupo de doença conforme CID10. Resultados: O gasto total apurado foi de $R \$ 43,7$ milhões. Dentre os medicamentos com maior gasto, observou-se o fator IX recombinante, utilizado para tratamento de hemofilia, como maior responsável $(22,53 \%)$, seguido da alfaglicosidase $(9,74 \%)$, do fingolimode $(8,44 \%)$ e da abiraterona $(6,63 \%)$. As doenças com maior demanda de atendimento via judicial foram as doenças do sangue que incluem as hemofilias $(26,6 \%)$, as neoplasias $(24,9 \%)$ e as doenças metabólicas $(17,5 \%)$. Conclusão: Os resultados obtidos permitiram verificar um padrão de demandas particular do DF, com uma participação importante no orçamento destinado à compra de medicamentos.

Palavras-chave: Judicialização. Medicamento. Gasto em saúde. Tecnologias em saúde

ABSTRACT: Judicialization as a phenomenon for right to health guarantee is an issue of increasing discussion in Brazil due to the constitutional definition of health in the country, which opens scenario for integrality. Objective: The way the lawsuits come impacting on public health policies in the three government levels puts this item as pressing point in the discussion of an agenda for the health system. Methods:This study analyzed the profile of public health expenditure in the Federal District with non-protocol drugs in order to understand in which circumstances the lawsuits of drugs ocurr in that territory. Administrative records of drug distribution were collected from September / 2014 to August / 2016. Results: The recorded expenditure was classified by item and by disease group as ICD-10. It was found that the total calculated expense was $\mathrm{R} \$ 43.7$ million, with recombinant factor IX, used

\footnotetext{
1 Doutor em Ciências da Saúde pela Universidade de Brasília, Farmacêutico, Coordenador do Centro de Avaliação de Tecnologias em Saúde, Hospital de Base do Distrito Federal, Brasília, Brasil. E-mail: ms.everton@gmail.com

${ }^{2}$ Doutora em Patologia Molecular pela Universidade de Brasília, Farmacêutica,Núcleo de Farmácia Ambulatorial Judicial, Diretoria de Assistência Farmacêutica, Secretaria de Estado de Saúde do Distrito Federal, Brasília, Brasil. E-mail: keylacaroline.a@gmail.com

${ }^{3}$ Farmacêutica-Bioquímica pela Universidade Federal de Minas Gerais, Diretoria de Assistência Farmacêutica, Secretaria de Estado de Saúde do Distrito Federal, Brasília, Brasil. E-mail: glauciasilveiracarvalho@gmail.com
} 
to treat hemophilia, as most responsible $(22.53 \%)$, followed by alpha-glucosidase $(9.74 \%)$, of fingolimod (8.44\%) and abiraterone (6.63\%). Diseases with increased demand for judicial services were blood diseases that include hemophilia (26.6\%), cancer (24.9\%) and metabolic disorders (17.5\%). Conclusión: The results obtained showed a pattern of particular demands of the Federal District, with an important role in drug purchasing budget.

Keywords: Judicialization. Drugs. Health expenditure. Health technology

RESUMEN: La judicialización como un fenómeno de garantizar el derecho social à la salud es un tema de creciente debate en Brasil debido a la definición constitucional de la salud en el país, que se abre el escenario para la integridad. Objetivo: La forma en que las demandas vienen impactando sobre las políticas de salud pública en los tres niveles de gestión pone este material como punto de discusión urgente de una agenda para el sistema de salud. Métodos: En este estudio se analizó el perfil del gasto en la salud pública en el Distrito Federal con medicamentos no estándar con el fin de entender cuáles son las circunstancias en que la judicialización se pasa en esto local. Fueron usados archivos administrativos de la distribución de medicamentos a partir de septiembre / 2014 a agosto / 2016. Resultados:El gasto registrado fue clasificado por tema y por grupo de enfermedades como la CIE-10. Se encontró que el gasto total calculado fue de $\mathrm{R} \$ 43,7$ millones de dólares. Entre los fármacos con un mayor gasto, se observó el factor IX recombinante, que se utiliza para tratar la hemofilia, como mayor responsable $(22,53 \%)$, seguido de la alfa-glucosidasa $(9,74 \%)$, del fingolimod $(8,44 \%)$ y de la abiraterona $(6,63 \%)$. Las enfermedades con mayor demanda de servicios judiciales fueron enfermedades de la sangre que incluyen la hemofilia (26,6\%), el cáncer $(24,9 \%)$ y los trastornos metabólicos $(17,5 \%)$. Conclusión: Los resultados obtenidos mostraron un estándar de demandas judiciales peculiar del Distrito Federal, con una parte importante del presupuesto para la compra de medicamentos.

Palabras-llave: Judicialización. Medicamento. Gasto en salud. Tecnologías en salud

\section{Introdução}

O crescente número de ações judiciais propostas em face do Poder Público com o fim de garantir o fornecimento de medicamentos e até mesmo a incorporação de novas tecnologias no âmbito do Sistema Único de Saúde - SUS tem sido motivo de preocupação para os gestores da saúde em todos os níveis federativos e vem sendo correntemente designada como "judicialização da saúde" (1), (2). As distorções decorrentes deste fenômeno, pelo menos no que diz respeito a medicamentos, são ocasionadas, sobretudo, pelo desconhecimento da política pública já implementada e muitas vezes decorrem de uma análise superficial da demanda, sem o devido amparo técnico (3).

O direito à saúde, aí compreendida a assistência farmacêutica, tem status de direito fundamental uma vez que de acordo com o artigo 196 da Constituição da República Federativa do Brasil: "A saúde é direito de todos e dever do estado, garantida mediante políticas sociais e econômicas, que visem à redução do risco de doenças e outros agravos 
e ao acesso universal e igualitário às ações e serviços para sua promoção, proteção e recuperação".

Em 19 de setembro de 1990, o Sistema Único de Saúde (SUS) foi regulamentado pela Lei $n^{\circ} 8.080$ que, dentre outros pontos, estabeleceu "assistência terapêutica integral, inclusive farmacêutica" e entre as ações, "a formulação da política de medicamentos (...) de interesse para a saúde (...)".

No campo da Assistência Farmacêutica no SUS, as ações estão definidas em três componentes: Componente Básico da Assistência Farmacêutica, que observa a oferta de medicamentos para o tratamento dos principais problemas de saúde da população em relação a sua demanda epidemiológica; Componente Estratégico da Assistência Farmacêutica, que objetiva disponibilizar medicamentos para o atendimento de Programas de saúde coordenados nacionalmente pelo Ministério da Saúde, de caráter transmissível e/ou alto impacto na saúde da população e Componente Especializado da Assistência Farmacêutica que é estratégia de acesso a medicamentos no âmbito do SUS, caracterizado pela busca da garantia da integralidade do tratamento medicamentoso, em nível ambulatorial, cujas linhas de cuidado estão definidas em Protocolos Clínicos e Diretrizes Terapêuticas publicados pelo Ministério da Saúde.

Para a disponibilização de medicamentos na rede pública de saúde esses devem passar pela etapa de seleção a qual pode culminar ou não na sua padronização. A padronização de um medicamento baseia-se na análise das melhores evidências clínicas disponíveis, bem como estudos de custo-efetividade e de impacto orçamentário. Esse processo é fundamental para a disponibilização de medicamentos eficazes, seguros e que mantenham a sustentabilidade do sistema de saúde (4). Este ponto se justifica pelo interesse coletivo, voltado ao atendimento da coletividade em detrimento da individualização do atendimento de saúde (5).

No âmbito da SES-DF, a incorporação dos medicamentos a serem fornecidos pela rede é avaliada pela Comissão Central de Farmácia e Terapêutica (CCFT), a qual funções e metodologia são estabelecidas através da Portaria no 10, de 28 de janeiro de 2016. A CCFT é órgão um deliberativo e multidisciplinar e dentre os seus objetivos estão: formular e implementar as políticas relacionadas com seleção de medicamentos nos diversos níveis de complexidade do sistema, assegurar prescrição e uso racional de medicamentos para 
garantir terapêutica eficaz e segura, além de melhoria na qualidade da assistência à saúde e o estabelecimento de critérios para o uso dos medicamentos selecionados.

Não obstante a todo o arcabouço legal em que se alicerça a Política Nacional de Medicamentos (PNM) bem como a Assistência Farmacêutica no âmbito da Saúde Pública no Distrito Federal, percebe-se que as decisões judiciais no campo das ações em que se pleiteiam medicamentos e tratamentos médicos em face dos entes públicos muitas vezes ainda são tomadas sem amparo na realidade concreta e técnica, sendo que em tais demandas presencia-se o aspecto emocional que subjaz à discussão jurídica, consistente na percepção do juiz que um cidadão jurisdicionado possa a vir a falecer "em suas mãos" por falta de um medicamento (6). Mas o dilema é bem mais amplo, como lembrado pelo eminente jurista Luís Roberto Barroso: "o que está em jogo, na complexa ponderação aqui analisada, é o direito à vida e à saúde de uns versus o direito à vida e à saúde de outros. Não há solução juridicamente fácil nem moralmente simples nessa questão" (14)

Neste contexto, esse estudo visou realizar uma análise sobre o gasto com medicamentos judicializados no âmbito do Sistema Único de Saúde, no território do Distrito Federal. A relevância dessa caracterização se dá pela necessidade de conhecer o perfil do gasto em saúde com medicamentos que impactam diretamente no planejamento e na gestão da assistência farmacêutica.

\section{Metodologia}

Este estudo foi realizado como um modelo de pesquisa documental, qualitativo e quantitativo.

O levantamento dos registros de compras por via judicial no Distrito Federal, foi feito por intermédio da Secretaria de Estado de Saúde do Distrito Federal, em que foram coletados os dados pelo sistema informatizado de gestão de materiais. O período compreendido na pesquisa foi de setembro/2014 a agosto/2016.

Para geração dos relatórios de medicamentos adquiridos por via judicial efetivamente distribuídos no Sistema Único de Saúde (SUS), foi utilizada a opção de relatório de consumo consolidado, com filtro para o grupo de medicamentos de interesse, classificados no sistema como medicamentos cadastrados de compra específica. Nesse caso é possível obter quais medicamentos foram distribuídos à população com aquisição específica por força de decisão judicial, sem limitações que poderiam surgir caso a base de dados tivesse como variável 
primária as compras homologadas ou empenhadas. Portanto, a seleção de consumo de medicamentos visou garantir a contagem daqueles tratamentos requeridos e que realmente foram fornecidos ao demandante.

Não foram consideradas nessa análise as demandas judiciais cujo objeto foram medicamentos padronizados pela Relação de Medicamentos Essenciais do DF, considerando que, em regra, o cumprimento das respectivas decisões judiciais é realizado com o estoque disponível pelas compras programadas a partir do consumo normal da rede de saúde local.

Para classificação do gasto com medicamento de acordo com a indicação terapêutica, foi utilizada a Classificação Estatística Internacional de Doenças e Problemas Relacionados à Saúde (CID-10).

Foi realizada estatística descritiva qualitativa dos dados obtidos, com cálculo das medidas de frequência para os gastos por medicamento e por doença.

Esse estudo não utilizou quaisquer dados de pacientes. Foram analisados registros administrativos de caráter contábil-financeiro.

\section{Resultados e Discussão}

O SUS propicia aos seus usuários medicamentos padronizados gratuitos de acordo com protocolos clínicos e diretrizes. Porém existem medicamentos que não estão contemplados na Relação Nacional de Medicamentos Essenciais (Rename) (7) e na Relação de Medicamentos Essenciais do Distrito Federal (Reme-DF) (8) dessa forma não são oferecidos regularmente em seu dispensário. Em resposta a isso, pacientes acionam o poder público judicialmente a fim de obrigá-lo a prover esses medicamentos não padronizados, endossando a judicialização da saúde no Brasil (5),(9).

Nesse sentido, o objeto do estudo consistiu na análise dos medicamentos não padronizados, denominados também como medicamentos de compra específica, que representam uma grande parcela dos medicamentos em litígio nas cortes. Utilizando a estratégia de busca de registros administrativos sobre a aquisição de medicamentos de compra específica por via judicial pelo SUS-DF, foram encontrados 244 medicamentos em 24 meses, no período de setembro/2014 a agosto/2016. O valor total gasto na aquisição desses medicamentos foi de $\mathrm{R} \$ 43.700 .727,04$ durante o período analisado. $\mathrm{A}$ representatividade desse volume de recursos pode ser mensurada a partir de outros relatos 
na literatura, como no estado de São Paulo, onde foi levantado que, em 2006, 77\% dos medicamentos solicitados por via judicial não pertenciam ao elenco de medicamentos fornecidos pela Secretaria Estadual de Saúde ou pelo Ministério da Saúde (10). No estado de Minas Gerais também foi possível observar que em 75,5\% dos casos avaliados os medicamentos solicitados por processos judiciais durante o período de 2005 à 2006, não pertenciam ao elenco padronizado da Rename e 56,7\% não estavam contemplados em programas da SES/MG (11).

Esses fatos são particularmente importantes no que diz respeito principalmente aos medicamentos não padronizados de alto custo, para os quais as despesas com o tratamento de um único indivíduo podem ser extremamente elevadas em detrimento dos gastos com a saúde coletiva (9). Nesse aspecto, a judicialização da saúde pode comprometer a execução de políticas de saúde no âmbito do SUS, uma vez que o cumprimento de determinações judiciais para o fornecimento de medicamentos, insumos e serviços de saúde acarreta gastos elevados e não programados (12).

Em uma análise dos medicamentos que mais contribuíram nesse montante, foi realizada uma curva $A B C$ (tabela 1), onde foi verificado que trinta medicamentos foram responsáveis pelo gasto de $R \$ 39.121 .099,74$ pelo SUS-DF, representando $89,52 \%$ do total gasto com os duzentos e quarenta e quatro itens judicializados com pelo SUS-DF no período de 24 meses (figura 1). 
Figura 1. Percentual do gasto com medicamentos por via judicial, classificado por medicamento, no período de setembro/2014 a agosto/2016.

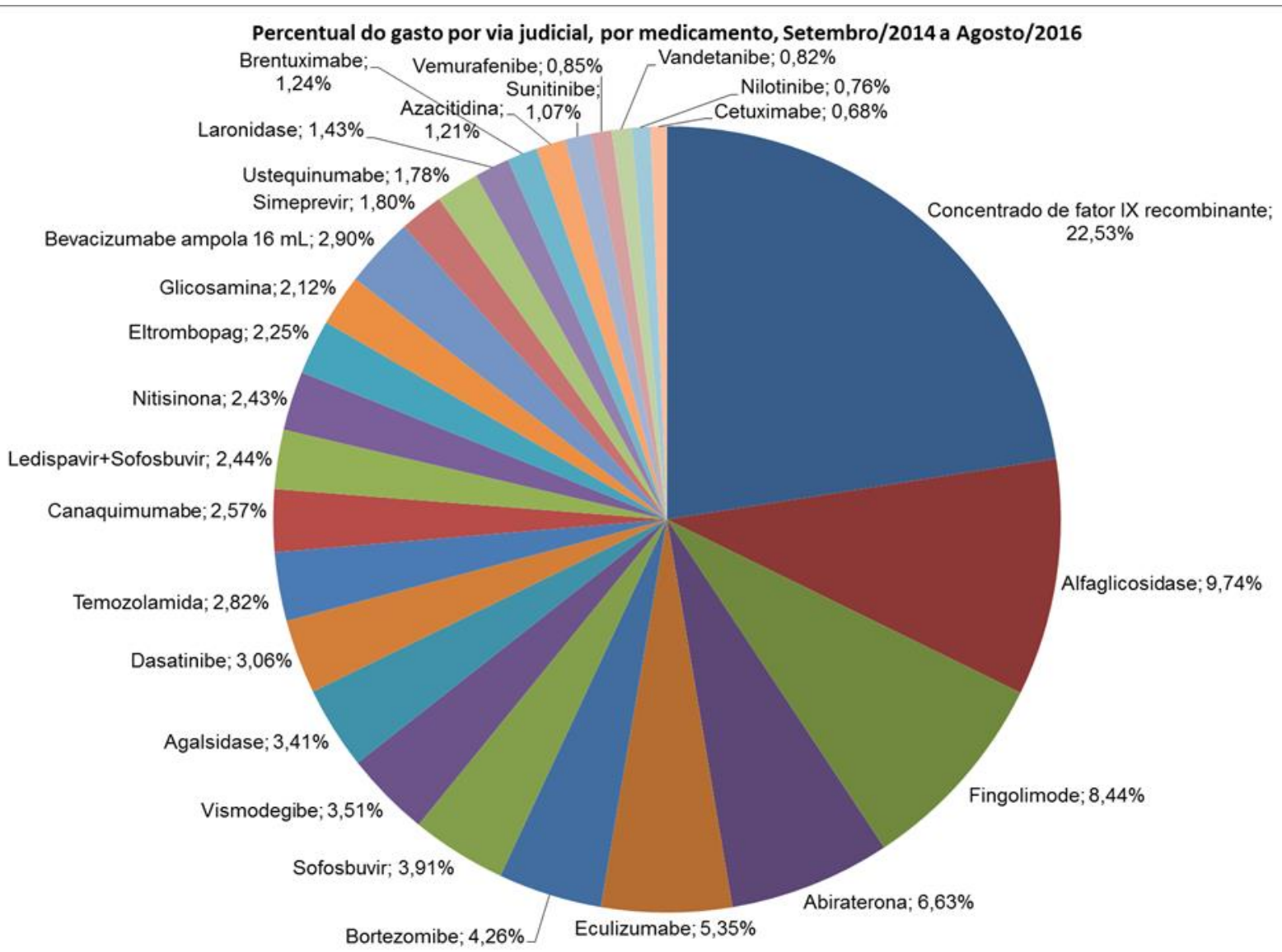

Foram alocados no mesmo grupo os medicamentos com apresentações diferentes, porém com mesmo princípio ativo.

Fonte: Elaboração própria s partir do Sistema informatizado de gestão de materiais da SES-DF

Dentre os trinta medicamentos que representam o maior custo para o SUS-DF (tabela 1), aproximadamente 17\% não apresentam registro na Agência Nacional de Vigilância Sanitária (Anvisa). Um valor de pelo menos três vezes acima quando comparado ao observado entre 2005 e 2006 no estado de Minas Gerais, no qual apenas 4,8\% não apresentava registro na Anvisa (12). Esse dado contraria os objetivos da PNM, uma vez que a mesma foi elaborada e implementada no país para garantir o acesso da população a medicamentos eficazes, seguros e de qualidade, promovendo assim seu uso racional. Cabe 
ressaltar que o uso de medicamentos sem registro sanitário ou fora das indicações para as quais foram registrados (off label) pode significar riscos à saúde do paciente (4).

Tabela 1. Valor gasto com medicamentos não-padronizados via demanda judicial no SUSDF, setembro/2014 a agosto/2016.

\begin{tabular}{|c|c|}
\hline 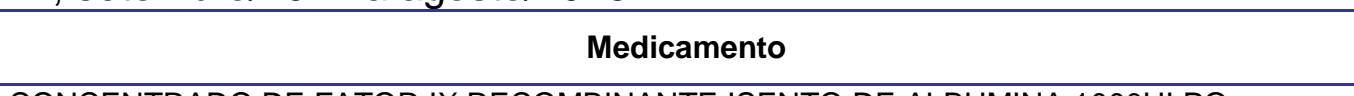 & $\begin{array}{c}\text { Gasto por } \\
\text { medicamento }(R \$)\end{array}$ \\
\hline $\begin{array}{l}\text { CONCENTRADO DE FATOR IX RECOMBINANTE ISENTO DE ALBUMINA 1000UI PO } \\
\text { LIOFILIZADO PARA SOLUÇAO INJETAVEL FRASCO AMPOLA + DILUENTE }\end{array}$ & $4.873 .323,40$ \\
\hline ALFALGLICOSIDASE PO LIOFILO INJETAVEL 50 MG FRASCO-AMPOLA & $3.809 .495,76$ \\
\hline FINGOLIMODE (CLORIDRATO) 0,5MG CÁPSULA & $3.303 .239,44$ \\
\hline ABIRATERONA (ACETATO) COMPRIMIDO 250 MG & $2.595 .286,19$ \\
\hline $\begin{array}{l}\text { CONCENTRADO DE FATOR IX RECOMBINANTE ISENTO DE ALBUMINA 500UI PO } \\
\text { LIOFILIZADO PARA SOLUÇAO INJETAVEL FRASCO AMPOLA + DILUENTE }\end{array}$ & $2.286 .284,38$ \\
\hline $\begin{array}{l}\text { ECULIZUMAB SOLUÇAO INJETAVEL } 300 \text { MG (10 MG/ML) FRASCO AMPOLA } 30 \text { ML } \\
\text { (IMPORTADO) }\end{array}$ & $2.093 .954,64$ \\
\hline BORTEZOMIBE PO LIOFILIZADO PARA SOLUCAO INJETAVEL 3,5MG FRASCO-AMPOLA & $1.666 .061,54$ \\
\hline $\begin{array}{l}\text { CONCENTRADO DE FATOR IX RECOMBINANTE ISENTO DE ALBUMINA 250UI PO } \\
\text { LIOFILIZADO PARA SOLUÇAO INJETAVEL FRASCO AMPOLA + DILUENTE }\end{array}$ & $1.652 .914,29$ \\
\hline SOFOSBUVIR COMPRIMIDO 400MG & $1.530 .692,11$ \\
\hline VISMODEGIBE CAPSULA 150 MG & $1.374 .330,38$ \\
\hline AGALSIDASE BETA PO LIOFILIZADO PARA INFUSAO INJETAVEL 35MG FRASCO AMPOLA & $1.334 .222,40$ \\
\hline DASATINIBE COMPRIMIDO REVESTIDO 50 MG & $1.195 .639,20$ \\
\hline TEMOZOLAMIDA CAPSULA 100MG & $1.103 .198,24$ \\
\hline CANAQUINUMABE PO PARA SOLUÇÃO INJETÁVEL 150 MG FRASCO-AMPOLA & $1.005 .275,65$ \\
\hline LEDISPAVIR + SOFOSBUVIR (90MG + 400MG) COMPRIMIDO & $953.299,40$ \\
\hline NITISINONE CAPSULA 10 MG (IMPORTADO) & $951.412,66$ \\
\hline ELTROMBOPAG OLAMINA COMPRIMIDO REVESTIDO 50MG & $879.794,66$ \\
\hline GLICOSAMINA (SULFATO) PO GRANULADO 1,5 G SACHE & $827.996,84$ \\
\hline BEVACIZUMABE SOL.INJETAVEL 25MG/ML FRASCO AMPOLA 16ML & $822.442,64$ \\
\hline SIMEPREVIR CAPSULA $150 \mathrm{MG}$ & $704.838,58$ \\
\hline $\begin{array}{l}\text { USTEQUINUMABE 45MG/0,5ML SOLUÇÃO INJETÁVEL FRASCO-AMPOLA OU SERINGA } \\
\text { PREENCHIDA }\end{array}$ & $697.080,07$ \\
\hline LARONIDASE SOLUCAO INJETAVEL 2,9MG/5ML FRASCO-AMPOLA & $559.210,70$ \\
\hline BRENTUXIMAB VEDOTIN 50MG INJETAVEL FRASCO OU FRASCO AMPOLA & $485.223,55$ \\
\hline $\begin{array}{l}\text { AZACITIDINA PO LIOFILIZADO PARA SUSPENSAO INJETAVEL FRASCO-AMPOLA 100MG } \\
\text { (VIA SC) }\end{array}$ & $472.606,55$ \\
\hline SUNITINIBE (MALATO) CAPSULA 50MG & $418.283,60$ \\
\hline VEMURAFENIBE COMPRIMIDO 240 MG & $332.516,80$ \\
\hline VANDETANIBE COMPRIMIDO REVESTIDO 300 MG & $319.183,20$ \\
\hline BEVACIZUMABE SOLUCAO INJETAVEL 25MG/ML FRASCO AMPOLA 4,0ML & $310.342,46$ \\
\hline NILOTINIBE CAPSULA GELATINOSA DURA 200MG & $296.952,32$ \\
\hline CETUXIMABE SOLUÇAO INJETAVEL 500 MG FRASCO-AMPOLA & $265.998,09$ \\
\hline Total & $39.121 .099,74$ \\
\hline
\end{tabular}

Fonte: Elaboração própria s partir do Sistema informatizado de gestão de materiais da SES-DF 
De acordo com o período avaliado, o medicamento responsável pelo maior gasto pela SES-DF foi o concentrado de fator IX recombinante $1000 \mathrm{UI}$, com $\mathrm{R} \$$ 4.873.323,40 (Tabela 1). Seu uso é indicado para tratamento de hemofilia B. Somando-se a apresentação de 500 UI, que isolada representou um gasto de $R \$ 2.286 .284,38$, e a apresentação de 250 UI, que contribuiu com um gasto de $\mathrm{R} \$ 1.652 .914,29$, houve um gasto total aproximado de 8,8 milhões de reais com esse único fármaco, que compreende 22,53\% do total (Figura 1).

Conforme dados do Ministério da Saúde, em 2014 havia 59 casos de hemofilia B no Distrito Federal, com uma prevalência de 1,5 casos/ 35.000 homens. Essa prevalência é considerada elevada no contexto nacional e estima-se ainda um sub-registro de 33,5\% (13). Assumindo que o tratamento de todos os pacientes tenha sido judicializado e considerando que em 2015 foram gastos cerca de $R \$ 4$ milhões com a aquisição judicial desses medicamentos, a razão de gasto anual por paciente seria da ordem de $\mathrm{R} \$ 68$ mil. Esse valor pode ser ainda maior, considerando o dado público da medida cautelar protocolada pelo Distrito Federal e deferida em 27 de julho de 2016 pelo Supremo Tribunal Federal (14). Essa medida relata a existência de nove ações judiciais contra o Distrito Federal até 2015, para o fornecimento de concentrado de fator IX recombinante. Nesse caso a razão de gasto anual por paciente seria da ordem de aproximadamente $R \$ 445$ mil. É ainda importante ressaltar que, no caso do fator IX recombinante, existe alternativa terapêutica padronizada pelo Ministério da Saúde, o fator IX plasmático, obtido por fracionamento de "pool" de plasma (15), e regularmente distribuído pelo SUS. Em que pese o programa de aquisição e distribuição do tratamento das hemofilias ser realizado pelo Ministério da Saúde, a judicialização nesse caso é um exemplo claro de um distúrbio do financiamento da saúde, com deslocamento de uma competência da União para o Distrito Federal.

Como segundo item com maior gasto no período, foram utilizados $\mathrm{R} \$ 3.809 .495,76$ para aquisição de alfaglicosidase, uma enzima necessária para reposição em indivíduos com doença metabólica. Na sequência, para o gasto com fingolimode, utilizado no tratamento de esclerose múltipla remitente recorrente, foi apurado um total de $R \$ 3.303 .239,44$. É interessante notar que, em todo o ano de 2015 houve um consumo de 11.800 unidades do medicamento nessa modalidade, com um valor de $R \$ 2.153 .732,50$. De janeiro a agosto de 2016, o quantitativo dispensado através de ação judicial foi de 3.500 unidades, com $R \$$ $637.198,97$ empregados. Essa redução no ritmo de aquisições judiciais do fingolimode provavelmente tem como uma das causas sua incorporação ao SUS em junho de 2014, 
conforme Protocolos Clínicos de Diretrizes Terapêuticas e análise prévia da Comissão Nacional de Incorporação de Tecnologias em Saúde no SUS.

Essa mesma tendência de queda possivelmente ocorrerá no valor observado para a compra de antirretrovirais para o tratamento de hepatites virais nos anos subsequentes. Durante o período analisado foram adquiridos por via judicial os medicamentos sofosbuvir $(R \$ 1.530 .692,11)$, ledispavir + sofosbuvir $(R \$ 953.299,40)$, simeprevir $(R \$ 704.838,58)$, telaprevir $\mathrm{R} \$ 194.697,75)$ e veruprevir + ombistavir + ritonavir + dasabuvir $(\mathrm{R} \$ 145.370,40)$ totalizando um gasto aproximado de 3,5 milhões de reais. Essa possibilidade deve-se ao fato de que algumas dessas novas terapias orais vêm sendo recentemente incorporadas ao SUS (16), ampliando o acesso regular e programático.

Diferentemente, no caso da glicosamina é possível esperar um movimento contrário.

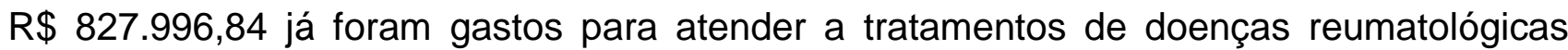
inflamatórias com uso de Glicosamina em paciente judicializados. Esse medicamento foi despadronizado durante o período analisado, possivelmente acarretando a ocorrência de compras específicas devido à demanda judicial. Atualmente, a via de obtenção desse produto pelo SUS-DF necessariamente passa pelo processo judicial. Nesse caso, 0 monitoramento do custo da compra também pode ser um termômetro interessante para avaliação do benefício a partir da mudança nessa política pública.

Analisando os achados de um estudo similar, Boing et al. (17) observaram que os medicamentos que mais demandaram recursos do Estado de Santa Catarina para aquisição judicial foram a laronidase, a temozolamida e o infliximab, com cerca de $R \$ 81$ mil, $R \$ 35$ mil e $R \$ 18$ mil gastos, respectivamente, entre os anos 2000 e 2006 . No presente estudo, o gasto com temozolamida representou $R \$ 1.103 .198,24$ e para laronidase foram $R \$$ $559.210,70$, ainda que esses medicamentos estejam fora dos dez maiores gastos com medicamentos não-padronizados judicializados.

$\mathrm{Na}$ análise do percentual de participação dos trinta principais itens no gasto total (Figura 1), nota-se que os três primeiros itens, fator IX recombinante, alfaglicosidase e fingolimode, apresentaram uma contribuição de $22,53 \%$, 9,74\% e 8,44\%, respectivamente. Se considerarmos também a abiraterona, com $6,63 \%$, e o eculizumabe, com $5,35 \%$, mais de $52 \%$ do gasto apurado é atribuível a esses cinco medicamentos não-padronizados, nos 24 meses analisados. A intervenção judicial no âmbito da gestão do setor de saúde apresentase como um fenômeno multifacetado com características específicas que vem exigindo uma 
atuação diferenciada do gestor público para responder a essas decisões judiciais no sentido de se evitar o crescimento de novas demandas e preservar os princípios e as diretrizes do SUS (4). Nesse sentido torna-se estratégica a busca pelo entendimento do perfil das demandas judiciais relacionadas ao uso dos medicamentos de uma determinada região, inclusive no desenvolvimento de políticas públicas.

Também foi possível observar que os produtos são adquiridos majoritariamente pela fonte de recursos hospitalar. Essa particularidade possivelmente é explicada pela necessidade de utilização da fonte de recursos exclusiva do DF, sem dotação orçamentária que inclua recursos federais. A alocação desse último recurso estaria contrariando a finalidade previamente definida, uma vez que a aquisição de medicamentos devido à judicialização não está contemplada em ações programáticas do orçamento público. A PNM determina as responsabilidades de cada esfera do governo no âmbito da assistência farmacêutica e do financiamento do SUS e o processo de judicialização da saúde desconsidera essa normatização, resultando prejuízo ao princípio de equidade em saúde (12).

No mapeamento dos valores por grupo de doenças (tabela 2), mais de $94 \%$ dos medicamentos obtidos na coleta de dados, entre 2014 e 2016, foram classificados conforme a indicação de uso, usando a Classificação Estatística Internacional de Doenças e Problemas Relacionados à Saúde (CID-10). A não classificação dos demais 6\% conforme essa classificação deve-se ao fato de que o uso para mais de uma indicação possível, contemplando mais de um grupo, não permite sua alocação de forma assertiva. É possível observar uma distribuição com maior volume de recurso utilizado para as hemofilias, que compreendeu $\mathrm{R} \$ 11.615 .582,26$.

Em seguida, nota-se o gasto de $\mathrm{R} \$ 10.875 .685,03$ com medicamentos não padronizados pela SES-DF para o tratamento de neoplasias. Os medicamentos oncológicos foram responsáveis por aproximadamente $24,9 \%$ do gasto com medicação não padronizada demandada via judicial durante esse período, sendo o medicamento abiraterona, indicado para o tratamento de câncer de próstata avançado metastático em população específica, responsável pelo gasto de $\mathrm{R} \$ 2.595 .286,19$ no período analisado, representando $6,63 \%$ do montante gasto com medicamentos judicializados pelo SUS-DF. Em análise do município de São Paulo, foi encontrado um percentual de $22 \%$ de medicamentos judicializados para tratamento de câncer (18). 
Em relação aos medicamentos oncológicos, salienta-se que os procedimentos de quimioterapias da Tabela de Procedimentos, Medicamentos e OPM do SUS, que define valores de ressarcimento ao serviço de saúde, não especificam nenhum medicamento a ser utilizado. Os esquemas antineoplásicos constam somente nos protocolos publicados. Desse modo, como exemplo, tem-se o bortezomibe, que representou $4,26 \%$ do gasto total apurado. Esse medicamento pode ser incluído no esquema de quimioterapia do mieloma múltiplo. A média dos preços de compras públicas informadas na base de dados do Banco de Preços em Saúde/Ministério da Saúde é de $R \$ 1.685,00$. O preço unitário estimado no SUS-DF é de cerca de $R \$ 2.100,00$ e nesse contexto, possivelmente a padronização do medicamento poderia reduzir o valor pago em negociação. Adicionalmente, o procedimento de quimioterapia de segunda linha de tratamento disponível na tabela de procedimentos do SUS permite um ressarcimento ao serviço de $\mathrm{R} \$ 1.715,60$.

Seguindo a sequência de doenças com maior contribuição do gasto têm-se as doenças endócrinas, nutricionais e metabólicas, principalmente síndromes metabólicas, doenças do sistema nervoso, as hepatites virais e doenças do sistema osteomuscular e do tecido conjuntivo. No caso desse último grupo, é importante salientar que somente um medicamento, a glicosamina, foi responsável pelo gasto observado.

Em estudo realizado em Minas Gerais, analisando-se o período de 2005 e 2006, foi observado que as doenças com mais medicamentos solicitados foram artrite reumatoide, diabetes mellitus e hipertensão arterial (12). A diferença de escopo comparada à nossa análise pode ser atribuída primeiramente ao fato de que os medicamentos imunobiológicos atualmente estão padronizados no elenco do DF, bem como ocorre com as insulinas de longa e curta duração, que são aquelas com maior potencial de demanda judicial. Diante da presença desses itens na lista já disponível a nível local, a curva de demandas se desloca para aqueles medicamentos não incluídos dentre as opções regulares.

$\mathrm{Na}$ perspectiva de avaliar a participação da compra de medicamentos nãopadronizados por decisão judicial no total da execução orçamentária com medicamentos no DF, verificou-se que em 2015 a judicialização levou a um gasto maior que 23 milhões. O total empenhado no mesmo período com a compra de medicamentos foi de cerca de 230 milhões e o total liquidado foi de 177 milhões, conforme dados do Sistema de Informações sobre Orçamentos Públicos em Saúde (19). Portanto, as compras judiciais representaram em torno de $10 \%$ da despesa empenhada e $13 \%$ da despesa efetivamente liquidada. Apesar 
do alto valor representado nos números apresentados, em outros estudos é possível observar uma razão de gasto com demandas judiciais de medicamentos bem mais acentuada. A preocupação nesse dado é a tendência de incremento acelerado com o tempo. Essa possibilidade pode ser exemplificada pelo aumento do gasto com a judicialização pelo Ministério da Saúde, passando de $R \$ 124,1$ milhões em 2010 para $R \$ 287,4$ milhões em $2012(3)$.

Em Santa Catarina, avaliou-se que em 2006 foram gastos $\mathrm{R} \$ 80$ milhões com assistência farmacêutica e cerca de $R \$ 21$ milhões para o cumprimento de decisões judiciais favoráveis à aquisição de medicamentos (17). A relação nesse caso é maior que $26 \%$. Essa diferença por ser explicada pelo fato de que neste estudo foi utilizada somente a base de dados de medicamentos não-padronizados.

O ingresso de processos judiciais contra o Poder Público é uma das formas que os cidadãos encontraram para garantir seus direitos de acesso ao medicamento ou tratamento específico, sendo considerada uma reivindicação legítima, pois objetiva garantir um direito fundamental (12). Porém é importante salientar que esse movimento de judicialização da saúde de forma descontrolada e irracional pode motivar o custeio e a disponibilidade de medicamentos sem considerar os princípios de segurança e eficácia da PNM, para uma pequena parcela da população, interferindo na efetividade da elaboração e execução das políticas públicas de saúde (4), (5).

\section{Conclusão}

Os resultados obtidos permitiram verificar um padrão de demandas particular do DF, com uma participação importante no orçamento destinado à compra de medicamentos.

Uma reflexão importante é avaliar até que ponto a garantia de acesso a esses medicamentos está proporcionando benefício esperado pelos indivíduos que demandam seu tratamento. Além disso, é necessário verificar se o plano terapêutico traçado pelo profissional prescritor também possui uma definição clara de quais as chances reais de atingir um desfecho clinicamente relevante e favorável. Em caso de fragilidade de alguma dessas premissas, o pleito de um medicamento não disponível por via regular não se sustentaria para justificar sua pertinência no processo de doença. Existe uma necessidade crescente no país de publicação de mais estudos para que se possa avaliar de forma 
adequada esse fenômeno recente da judicialização no âmbito da saúde pública, com intuito de melhor compreendê-la e consequentemente traçar estratégias.

\section{Referências}

1. Marchesini Stival SL, Girão F. A judicialização da saúde: breves comentários. Cad Ibero Am Direito Sanit (Impr). 2016;5(2):141-58.

2. Bittencourt GB. O Estado da Arte da produção acadêmica sobre o fenômeno da judicialização da saúde no Brasil. Cad Ibero Am Direito Sanit (Impr). 2016;5(1):102-21.

3. Balestra Neto O. A jurisprudência dos tribunais Superiores e o direito à saúde Evolução rumo à racionalidade. Rev direito sanit. 2015;16(1):87-111.

4. Pepe VLE, Figueiredo TdA, Simas L, Osorio-de-Castro CGS, Ventura M. A judicialização da saúde e os novos desafios da gestão da assistência farmacêutica. Ciênc saúde coletiva. 2010;15(5):2405-14.

5. Biehl J, Petryna A. Tratamentos jurídicos: os mercados terapêuticos e a judicialização do direito à saúde. Hist Cienc Saude Manguinhos. 2016;23(1):173-92.

6. Neto EHS, Castro GM. Direito Sanitário - Manifestações atuais: visão crítica de advogados de Estado. Brasília: Editora Kiron; 2012.

7. Brasil. Ministério da Saúde. Secretaria de Ciência TelEDdAFelE. Relação nacional de medicamentos essenciais: RENAME 2014. 9. ed. rev. atual. ed. Brasília: Ministério da Saúde; 2015. 230 p. p.

8. Relação de Medicamentos Essenciais do Distrito Federal 2016

[http://www.saude.df.gov.br/images/DIASF/2016/3__Medicamentos_padronizados_na_SES_DF.pdf].

9. Medeiros M, Diniz D, Schwartz IV. [The thesis of judicialization of health care by the elites: medication for mucopolysaccharidosis]. Cien Saude Colet. 2013;18(4):1079-88.

10. Chieffi ALBRB, Cadernos de Saúde P. Judicialização da política pública de assistência farmacêutica e equidade. Cad Saúde Pública. 2009;25(8):11.

11. Campos Neto OH, Acurcio FdA, Machado MAdÁ, Ferré F, Barbosa FLV, Cherchiglia $\mathrm{ML}$, et al. Médicos, advogados e indústria farmacêutica na judicialização da saúde em Minas Gerais, Brasil. Rev Saude Publica. 2012;46(5):784-90.

12. Machado MA, Acurcio FeA, Brandão CM, Faleiros DR, Guerra AA, Cherchiglia ML, et al. Judicialization of access to medicines in Minas Gerais state, Southeastern Brazil. Rev Saude Publica. 2011;45(3):590-8. 
13. Brasil. Ministério da Saúde. Secretaria de Atenção à Saúde. Departamento de Atenção Hospitalar e de Urgência. Coordenação-Geral de Sangue e H. Perfil das coagulopatias hereditárias no Brasil: 2014. Brasília: Editora do Ministério da Saúde; 2015. 62p. il. p.

14. Medida cautelar na suspensão de liminar 1.019 Distrito Federal (2016).

15. Brasil. Ministério da Saúde. Secretaria de Atenção à Saúde. Departamento de Atenção Especializada. Coordenção da Política Nacional de Sangue e H. Manual de tratamento das coagulopatias hereditárias. Brasília: Editora do Ministério da Saúde; 2006. 76 p. p.

16. Portaria SCTIE/MS n² 29, de 22 de junho de 2015., (2015).

17. Boing A, Bloemer NS, Roeler C, Fernandes S. A judicialização do acesso aos medicamentos em Santa Catarina: um desafio para gestão do sistema de saúde. Rev direito sanit. 2013;14(1):82-97.

18. Vieira FS, Zucchi P. Distorções causadas pelas ações judiciais à política de medicamentos no Brasil. Rev Saúde Pública. 2007;41(2):9.

19. Brasil. Ministério da Saúde. Secretaria Exectutiva. Departamento de Economia da Saúde leD. Sistema de Informações sobre Orçamentos Públicos em Saúde Brasília: Ministério da Saúde; 2016 [http://portalsaude.saude.gov.br/index.php/oministerio/principal/siops/mais-sobre-siops/6116-dados-informados-estados-e-distritofederal].

Como citar este artigo:

Silva EM, Almeida KC, Pessôa GSC. Análise do gasto com judicialização de medicamentos no Distrito Federal, Brasil. Revista Cadernos Ibero-Americanos de Direito Sanitário. 2017 jan./mar, 6(1):112-126. 